\title{
Resonant Homoclinic Bifurcations with Orbit Flips and Inclination Flips"
}

\author{
Tiansi Zhang \\ College of Science, University of Shanghai for Science and Technology, Shanghai, China \\ Email: zhangts1209@163.com
}

Received December 17, 2012; revised January 17, 2013; accepted January 25, 2013

\begin{abstract}
Homoclinic bifurcation with one orbit flip, two inclination flips and resonance in the tangent directions of homoclinic orbit is considered. By studying the associated successor functions constructed from a local active coordinate system, we prove the existence of double 1-periodic orbit, 1-homoclinic orbit, and also some coexistence conditions of 1-periodic orbit and 1-homoclinic orbit.
\end{abstract}

Keywords: Orbit Flip; Inclination Flip; Resonance

\section{Introduction and Hypotheses}

Flips homoclinic bifurcations are comprehensively investigated during the last decade (see [1-10]), which produce complicated bifurcations, such as the saddlenode bifurcations, the period-doubling bifurcations and the homoclinic-doubling bifurcations.

Recently, the flip of heterodimensional cycle or accompanied by transcritical bifurcation is discussed much (see [11-13]). The double and triple periodic orbit bifurcation are proved to exist, and also some coexistence conditions for homoclinic orbits and periodic orbits. But their research is not focused on multiple flips since it is a interesting problem and full of challenges due to the high codimension and complexity. In this paper, we develop a study of resonant homoclinic bifurcation with one orbit flip and two inclination flips, where the resonance takes place in the tangent direction of the homoclinic orbit. This is a codimension- 4 problem, by using the local moving frame method established in [11,14,15], we get the existence of a double 1-periodic orbit, some 1-periodic orbits and 1-homoclinic orbits, and the coexistence conditions of 1-periodic orbits and 1-homoclinic orbits.

We consider the following two systems,

$$
\begin{aligned}
& \dot{z}=f(z)+g(z, \mu), \\
& \dot{z}=f(z),
\end{aligned}
$$

where $r \geq 3, z \in \mathbb{R}^{4}, \mu \in \mathbb{R}^{l}, l \geq 4,0<|\mu| \ll 1, \quad f(0)=0$, $g(0, \mu)=g(z, 0)=0$.

*Project supported by National Natural Science Foundation of China (Grant: 11126097) and by Scientific Research Foundation for the Returned Overseas Chinese Scholars, State Education Ministry.
Notice that system (1.2) is an unperturbed system of (1.1) and assume it has an orbit

$$
\Gamma=\{z=r(t): t \in \mathbb{R}, r( \pm \infty)=0\}
$$

homoclinic to the hyperbolic equilibrium $z=O$, which has two negative and two positive eigenvalues, $\lambda_{1}, \lambda_{2}$, $-\rho_{1},-\rho_{2}$, and $\lambda_{2}>\lambda_{1}>0>-\rho_{1}>-\rho_{2}$.

\section{Hypotheses}

Set $W^{s}$ (resp. $W^{s s}$ ) and $W^{u}$ (resp. $W^{u u}$ ) the stable (resp. strong stable) manifold and unstable (resp. strong unstable) manifold of the equilibrium $z=O$, respectively. We suppose that

(H1) $\lambda_{1}(\mu) \equiv \rho_{2}(\mu)$ for $|\mu| \ll 1$, where $\lambda_{1}(0)=\lambda_{1}$ and $\rho_{2}(0)=\rho_{2}$.

(H2) Define $e^{+}=\lim _{t \rightarrow-\infty} \dot{r}(t) /|\dot{r}(t)|, e_{s}^{-}=\lim _{t \rightarrow+\infty} \dot{r}(t) /|\dot{r}(t)|$, then $e^{+} \in T_{0} W^{u}$ and $e_{s}^{-} \in T_{0} W^{s s}$ are unit eigenvectors corresponding to $\lambda_{1}$ and $-\rho_{2}$ respectively, where $T_{0} W^{u}$ is the tangent space of the corresponding manifold $W^{u}$ at the saddle $z=O$, and the similar meaning for $T_{0} W^{s s}$

(H3) Denote by $e_{u}^{+}$and $e^{-}$the unit eigenvectors corresponding to $\lambda_{2}$ and $-\rho_{1}$ respectively, there are

$$
\begin{aligned}
& \lim _{t \rightarrow+\infty}\left\{T_{r_{i}(t)} W^{u}, T_{r_{i}(t)} W^{s}, e_{u}^{+}\right\}=\mathbb{R}^{4}, \\
& \lim _{t \rightarrow-\infty}\left\{T_{r_{i}(t)} W^{u}, T_{r_{i}(t)} W^{s}, e_{s}^{-}\right\}=\mathbb{R}^{4} .
\end{aligned}
$$

Remark 1.1 Hypotheses (H1) is a resonant condition, while (H2) - (H3) mean the homoclinic orbit has one orbit flip and two inclinations flips. 
The paper is organized as follows. In Section 2, we first transform system (1.1) into two normal forms, then construct a regular map in some neighborhood of the homoclinic orbit and a singular map in some neighborhood of the equilibrium respectively to establish the Poincaré map. In Section 3, we develop the bifurcation study through searching for solutions of the bifurcation equation. Finally a short conclusion about the flips bifurcation is given in Section 4 .

\section{Local Active Coordinate Frame and Poincaré Map}

We first give two normal forms of system (1.1) and then construct the Poincaré map. Firstly system (1.1) can be transformed into the following form in some neighborhood $U$ of the origin $O$ due to the theory of invariance manifolds, (refer to [14,15])

$$
\begin{aligned}
\dot{x} & =\left[\lambda_{1}(\mu)+a(\mu) x v+o(|x v|)\right] x \\
& +O(u)\left[O\left(x^{2} v\right)+O(y)\right], \\
\dot{y}= & {\left[-\rho_{1}(\mu)+b(\mu) x v+o(|x v|)\right] y } \\
& +O(v)[O(x y v)+O(\mu)], \\
\dot{u}= & {\left[\lambda_{2}(\mu)+c(\mu) x v+o(|x v|)\right] u } \\
& +x^{2} H_{1}(x, y, v), \\
\dot{v} & =\left[-\rho_{2}(\mu)+d(\mu) x v+o(|x v|)\right] v \\
& +y^{2} H_{2}(x, y, u),
\end{aligned}
$$

where $H_{2}(0, y, 0)=0, \quad \lambda_{1}(0)=\lambda_{1}, \quad \lambda_{2}(0)=\lambda_{2}$, $\rho_{1}(0)=\rho_{1}$ and $\rho_{2}(0)=\rho_{2} \cdot a(\mu), b(\mu), c(\mu)$ and $d(\mu)$ are parameters depending on $\mu$.

One may see that from (2.1), $\Gamma \cap W_{\text {loc }}^{u}$ and $\Gamma \cap W_{\text {loc }}^{s}$ are straightened locally to be the $x, v$ axes in neighborhood of $O$, so it is possible to take some time $T>0$ large enough, such that $r(-T)=\{\delta, 0,0,0\}$ and $r(T)=\{0,0,0, \delta\}$, where $\delta$ is small and

$$
\{(x, y, u, v):|x|,|y|,|u|,|v|<2 \delta\} \subset U .
$$

Now consider the linear variational system

$$
\dot{z}=D \boldsymbol{f}(r(t)) z,
$$

and its adjoint system

$$
\dot{z}=-(D f(r(t)))^{*} z .
$$

Matrix theory shows that system (2.2) has a fundamental solution matrix and furthermore it can be chosen as follows (refer to [11,14-15])

Lemma 2.1 There exists a fundamental solution matrix $\boldsymbol{Z}(t)=\left(z_{1}(t), z_{2}(t), z_{3}(t), z_{4}(t)\right)$ of system (2.2) satisfying

$$
\begin{aligned}
& \boldsymbol{Z}(-T)=\left(\begin{array}{cccc}
w_{11} & w_{21} & 0 & w_{41} \\
0 & 0 & 0 & w_{42} \\
w_{13} & 0 & 1 & w_{43} \\
w_{14} & 0 & 0 & w_{44}
\end{array}\right), \\
& \boldsymbol{Z}(T)=\left(\begin{array}{cccc}
0 & 0 & w_{31} & 0 \\
w_{12} & 0 & w_{32} & 1 \\
1 & 0 & w_{33} & 0 \\
0 & 1 & w_{34} & 0
\end{array}\right),
\end{aligned}
$$

where

$$
\begin{aligned}
& z_{1}(t) \in\left(T_{r(t)} W^{u}\right)^{c} \cap\left(T_{r(t)} W^{s}\right)^{c}, \\
& z_{2}(t)=-\dot{r}(t) /\left|\dot{r}^{y}(T)\right| \in T_{r(t)} W^{u} \cap T_{r(t)} W^{s}, \\
& z_{3}(t) \in T_{r(t)} W^{u}
\end{aligned}
$$

and $z_{4}(t) \in T_{r(t)} W^{s}$, and $w_{14} w_{21} w_{31} w_{42} \neq 0, w_{21}<0$.

Obviously $\left(\boldsymbol{Z}^{-1}(t)\right)^{*}$ is a fundamental solution matrix of system (2.3), denote by

$$
\Phi(t)=\left(\phi_{1}(t), \phi_{2}(t), \phi_{3}(t), \phi_{4}(t)\right)=\left(\boldsymbol{Z}^{-1}(t)\right)^{*} .
$$

We here introduce a new coordinate $N=\left(n_{1}, 0, n_{3}, n_{4}\right)^{*}$ and set

$$
\begin{aligned}
s(t) & =r(t)+\boldsymbol{Z}(t) N \\
& =r(t)+z_{1}(t) n_{1}+z_{3}(t) n_{3}+z_{4}(t) n_{4} .
\end{aligned}
$$

Naturally we can choose two cross sections of $\Gamma$, see Figure 1,

$$
\begin{aligned}
& S_{0}=\{z=s(T):|x|,|y|,|u|,|v|<2 \delta\} \subset U, \\
& S_{1}=\{z=s(-T):|x|,|y|,|u|,|v|<2 \delta\} \subset U .
\end{aligned}
$$

Substitute (2.4) into (1.1), there is

$$
\dot{n}_{i}=\phi_{i}^{*}(t) g_{\mu}(r(t), 0) \mu+\text { h.o.t., } i=1,3,4 .
$$

Integrating both sides from $-T$ to $T$, we further have

$$
n_{i}(T)=n_{i}(-T)+M_{i} \mu+\text { h.o.t. }, i=1,3,4,
$$

where

$$
\boldsymbol{M}_{1}=\int_{-T}^{T} \phi_{1}^{*}(t) g_{\mu}(r(t), 0) \mathrm{d} t=\int_{-\infty}^{+\infty} \phi_{1}^{*}(t) g_{\mu}(r(t), 0) \mathrm{d} t .
$$

Equation (2.5) defines indeed a map $F_{1}: S_{1} \rightarrow S_{0}$ in some tube region near $\Gamma$,

$$
\begin{aligned}
N(-T) & =\left(n_{1}(-T), 0, n_{3}(-T), n_{4}(-T)\right)^{*} \mapsto N(T) \\
& =\left(n_{1}(T), 0, n_{3}(T), n_{4}(T)\right)^{*}
\end{aligned}
$$

see Figure 1(a). If set 


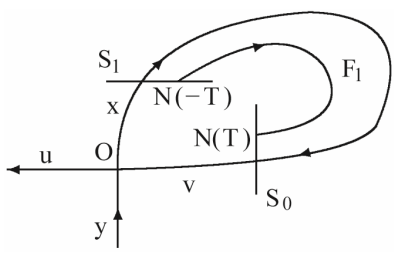

(a)

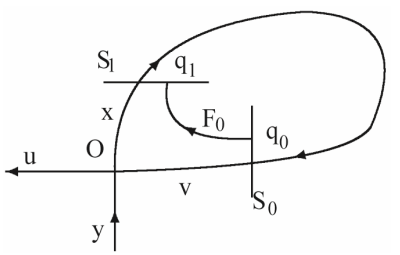

(b)
Figure 1. Transition maps. (a) $F_{1}: S_{1} \rightarrow S_{0}$; (b) $F_{0}: S_{0} \rightarrow S_{1}$.

$$
\begin{aligned}
q_{2 j} & =q_{2 j}\left(x_{2 j}, y_{2 j}, u_{2 j}, v_{2 j}\right) \in S_{0}, q_{2 j+1} \\
& =q_{2 j+1}\left(x_{2 j+1}, y_{2 j+1}, u_{2 j+1}, v_{2 j+1}\right) \in S_{1}
\end{aligned}
$$

and

$$
\begin{aligned}
& N_{2 j}\left(n_{2 j, 1}, 0, n_{2 j, 3}, n_{2 j, 4}\right), \\
& N_{2 j+1}\left(n_{2 j+1,1}, 0, n_{2 j+1,3}, n_{2 j+1,4}\right), j=0,1,2, \cdots
\end{aligned}
$$

we can obtain the following expressions,

$$
\begin{aligned}
n_{2 j, 1} & =u_{2 j}-w_{33} w_{31}^{-1} x_{2 j}, \\
n_{2 j, 3} & =w_{31}^{-1} x_{2 j}, \\
n_{2 j, 4} & =y_{2 j}-w_{12} u_{2 j}+\left(w_{12} w_{33}-w_{32}\right) w_{31}^{-1} x_{2 j}, \\
n_{2 j+1,1} & =w_{14}^{-1} v_{2 j+1}-w_{44} w_{14}^{-1} w_{42}^{-1} y_{2 j+1}, \\
n_{2 j+1,3} & =u_{2 j+1}-w_{13} w_{14}^{-1} v_{2 j+1} \\
& +\left(w_{13} w_{44} w_{14}^{-1}-w_{43}\right) w_{42}^{-1} y_{2 j+1}, \\
n_{2 j+1,4} & =w_{42}^{-1} y_{2 j+1},
\end{aligned}
$$

and

$$
x_{2 j+1} \approx \delta, v_{2 j} \approx \delta .
$$

Using the flow of system (2.1) in the neighborhood $U$, we can set up a map

$$
F_{0}: S_{0} \rightarrow S_{1} ; q_{0}\left(x_{0}, y_{0}, u_{0}, v_{0}\right) \mapsto q_{1}\left(x_{1}, y_{1}, u_{1}, v_{1}\right)
$$

defined as (see $[14,15]$ )

$$
\begin{aligned}
& x_{0}=x(T)=s^{\frac{\lambda_{1}(\mu)}{\rho_{1}(\mu)}} x_{1}+O\left(x_{1}^{2} v_{0} s^{\frac{2 \lambda_{1}(\mu)}{\rho_{1}(\mu)}} \ln s\right), \\
& y_{1}=y(T+\tau)=s y_{0}+O\left(x_{1} v_{0} y_{0} s^{\frac{\rho_{1}(\mu)+\lambda_{1}(\mu)}{\rho_{1}(\mu)}} \ln s\right), \\
& u_{0}=u(T)=s^{\frac{\lambda_{2}(\mu)}{\rho_{1}(\mu)}} u_{1}+O\left(x_{1} v_{0} u_{1} s^{\frac{\lambda_{1}(\mu)+\lambda_{2}(\mu)}{\rho_{1}(\mu)}} \ln s\right), \\
& v_{1}=v(T+\tau)=s^{\frac{\rho_{2}(\mu)}{\rho_{1}(\mu)}} v_{0}+O\left(x_{1} v_{0}^{2} s^{\frac{2 \rho_{2}(\mu)}{\rho_{1}(\mu)}} \ln s\right),
\end{aligned}
$$

where $s=\mathrm{e}^{-\rho_{1}(\mu) \tau}$ is the Silnikov time and $\tau$ is the time going from $q_{0}$ to $q_{1}$, see Figure 1(b).
From the above the Poincaré map

$$
F=F_{1} \circ F_{0}: q_{0} \in S_{0} \mapsto q_{0} \in S_{0}
$$

is obtained

$$
\begin{aligned}
n_{21} & =w_{14}^{-1} \delta s^{\frac{\rho_{2}(\mu)}{\rho_{1}(\mu)}}-w_{44} w_{14}^{-1} w_{42}^{-1} s y_{0}+M_{1} \mu+\text { h.o.t., } \\
n_{23} & =u_{1}-w_{13} w_{14}^{-1} \delta s^{\frac{\rho_{2}(\mu)}{\rho_{1}(\mu)}} \\
& +\left(w_{13} w_{44} w_{14}^{-1}-w_{43}\right) w_{42}^{-1} s y_{0}+\boldsymbol{M}_{3} \mu+\text { h.o.t., } \\
n_{24} & =w_{42}^{-1} s y_{0}+\boldsymbol{M}_{4} \mu+\text { h.o.t.. }
\end{aligned}
$$

Then the corresponding associated successor function $G\left(s, u_{1}, y_{0}\right)=\left(G_{1}, G_{3}, G_{4}\right)=F\left(q_{0}\right)-q_{0}$ is

$$
\begin{aligned}
G_{1}= & w_{14}^{-1} \delta s^{\frac{\rho_{2}(\mu)}{\rho_{1}(\mu)}}-u_{1} s^{\frac{\lambda_{2}(\mu)}{\rho_{1}(\mu)}}+w_{33} w_{31}^{-1} \delta s^{\frac{\lambda_{1}(\mu)}{\rho_{1}(\mu)}} \\
& -w_{44} w_{14}^{-1} w_{42}^{-1} s y_{0}+\boldsymbol{M}_{1} \mu+\text { h.o.t., } \\
G_{3}= & u_{1}-w_{13} w_{14}^{-1} \delta s^{\frac{\rho_{2}(\mu)}{\rho_{1}(\mu)}}-w_{31}^{-1} \delta s^{\frac{\lambda_{1}(\mu)}{\rho_{1}(\mu)}} \\
& +\left(w_{13} w_{44} w_{14}^{-1}-w_{43}\right) w_{42}^{-1} s y_{0}+\boldsymbol{M}_{3} \mu+\text { h.o.t., } \\
G_{4}= & w_{42}^{-1} s y_{0}-y_{0}+w_{12} s^{\frac{\lambda_{2}(\mu)}{\rho_{1}(\mu)}} u_{1} \\
& +\left(w_{32}-w_{12} w_{33}\right) w_{31}^{-1} \delta s^{\frac{\lambda_{1}(\mu)}{\rho_{1}(\mu)}}+\boldsymbol{M}_{4} \mu+\text { h.o.t.. }
\end{aligned}
$$

Since $s=\mathrm{e}^{-\rho_{1}(\mu) \tau}$ is defined by the flying time from the point in $S_{0}$ to $S_{1}$, obviously $s>0$ means $\tau$ is limited; $s=0$ means $\tau=+\infty$, which indicate the existence of a periodic orbit or a homoclinic orbit of system (1.1). So in the following section, we focus us on the solutions $s$ of (2.10).

\section{Bifurcation Results}

The last two equations in (2.10) give

$$
\begin{aligned}
& u_{1}=\left(w_{31}^{-1}+w_{13} w_{14}^{-1}\right) \delta s^{\frac{\lambda_{1}}{\rho_{1}}}-\boldsymbol{M}_{3} \mu+O\left(s y_{0}\right), \\
& y_{0}=\left(w_{32}-w_{33} w_{12}\right) w_{31}^{-1} \delta s^{\frac{\lambda_{1}}{\rho_{1}}}+\boldsymbol{M}_{4} \mu+O\left(s y_{0}\right)+O\left(s^{\frac{\lambda_{2}}{\rho_{1}}}\right) .
\end{aligned}
$$

Then from $G_{1}=0$ we get the bifurcation equation

$$
\begin{aligned}
F(s, \mu) \equiv & \left(1+w_{14} w_{33} w_{31}^{-1}\right) s^{\frac{\lambda_{1}}{\rho_{1}}}+\delta^{-1} w_{14} \boldsymbol{M}_{3} \mu s^{\frac{\lambda_{2}}{\rho_{1}}} \\
& -w_{44} w_{42}^{-1} \delta^{-1} \boldsymbol{M}_{4} \mu s+\delta^{-1} w_{14} \boldsymbol{M}_{1} \mu \\
& -w_{44} w_{42}^{-1}\left(w_{32}-w_{12} w_{33}\right) w_{31}^{-1} s^{\frac{\rho_{1}+\lambda_{1}}{\rho_{1}}} \\
& +\left(w_{13} w_{44}-w_{14} w_{43}\right) w_{42}^{-1} \delta^{-1} \boldsymbol{M}_{4} \mu s^{\frac{\rho_{1}+\lambda_{2}}{\rho_{1}}} \\
& + \text { h.o.t. }=0 .
\end{aligned}
$$

Notice that we have put higher orders terms into h.o.t. 
and omitted the parameter $\mu$ in the eigenvalues for concision.

Define two functions as

$$
\begin{aligned}
P(s, \mu) & =w_{44} w_{42}^{-1} \delta^{-1} \boldsymbol{M}_{4} \mu s-\delta^{-1} w_{14} \boldsymbol{M}_{1} \mu+\text { h.o.t., } \\
Q(s, \mu)= & \left(1+w_{14} w_{33} w_{31}^{-1}\right) s^{\frac{\lambda_{1}}{\rho_{1}}}+\delta^{-1} w_{14} \boldsymbol{M}_{3} \mu s^{\frac{\lambda_{2}}{\rho_{1}}} \\
& -w_{44} w_{42}^{-1}\left(w_{32}-w_{12} w_{33}\right) w_{31}^{-1} s^{\frac{\rho_{1}+\lambda_{1}}{\rho_{1}}}+\text { h.o.t.. }
\end{aligned}
$$

Indeed here $F(s, \mu)=Q(s, \mu)-P(s, \mu)$. By analysis of the curves $f=P(s, \mu)$ and $f=Q(s, \mu)$, one may immediately get the following statements.

Theorem 3.1 Suppose that $\operatorname{Rank}\left(\boldsymbol{M}_{1}, \boldsymbol{M}_{4}\right)=2$, then in the region $R_{11} \cup R_{12}$, system (1.1) has a unique 1-periodic orbit near $\Gamma$; in the region $R_{21}$, system (1.1) has not any 1-periodic orbit.

Proof Because

$$
\begin{aligned}
& P(0, \mu)=-w_{14} \delta^{-1} \boldsymbol{M}_{1} \mu+\text { h.o.t., } Q(0, \mu)=0, \\
& P^{\prime}(s, \mu)=w_{44} w_{42}^{-1} \delta^{-1} \boldsymbol{M}_{4} \mu+\text { h.o.t., } \\
& Q^{\prime}(s, \mu)=\frac{\lambda_{1}}{\rho_{1}}\left(1+w_{14} w_{33} w_{31}^{-1}\right) s^{\frac{\lambda_{1}}{\rho_{1}}-1}+\text { h.o.t., }
\end{aligned}
$$

the curve $f=Q(s, \mu)$ has no inflexion point, so th $\mathrm{e}$ line $f=P(s, \mu)$ and the curve $f=Q(s, \mu)$ must intersect at a unique point in the region $R_{11} \cup R_{12}$, where

$$
R_{11}=\left\{\mu \mid w_{14} \boldsymbol{M}_{1} \mu<0, w_{42} w_{44} \boldsymbol{M}_{4} \mu<0\right\}
$$

and

$$
R_{12}=\left\{\mu \mid w_{14} \boldsymbol{M}_{1} \mu<0, w_{42} w_{44} \boldsymbol{M}_{4} \mu>0\right\},
$$

see Figure 2(a). Namely there exists a point $s>0$ such that

$$
F(s, \mu)=Q(s, \mu)-P(s, \mu)=0,
$$

therefore system (1.1) has a unique 1-periodic orbit. On the contrary, there is not such a intersection point in the region

$$
R_{21}=\left\{\mu \mid w_{14} \boldsymbol{M}_{1} \mu>0, w_{42} w_{44} \boldsymbol{M}_{4} \mu<0\right\},
$$

see Figure 2(b).

Theorem 3.2 Suppose that $\operatorname{Rank}\left(\boldsymbol{M}_{1}, \boldsymbol{M}_{4}\right)=2$, then in the region $R_{22}$, system (1.1) has a unique double 1periodic orbit near $\Gamma$ located in the bifurcation surface $S N^{1}$.

Moreover when $\mu$ lies on the side of $S N^{1}$ pointing to the (resp. opposite) direction $-\left(\operatorname{sgn} w_{14}\right) \boldsymbol{M}_{1}$, system (1.1) has two (resp. not any) 1-periodic orbits near $\Gamma$.

Proof We know that the existence of a double 1-periodic orbit corresponds to a double solution $s^{*}$ of (3.1). According to the proof of Theorem 3.1, it is enough to search the tangent point of the curves $f=P(s, \mu)$ and

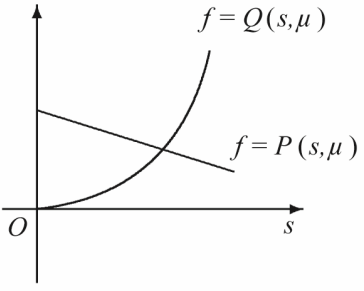

(a)

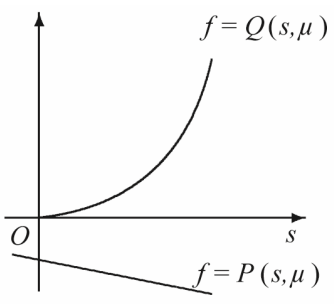

(b)

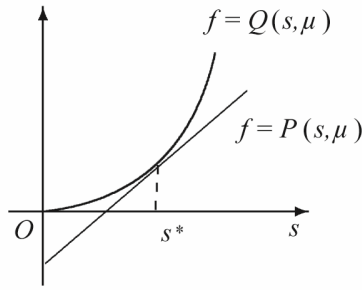

(c)

Figure 2. Location between the curves. (a) $\mu \in R_{11} \cup R_{12}$; (b) $\mu \in R_{21}$; (c) $\mu \in R_{22}$.

$f=Q(s, \mu)$, that is to solve

$$
P(s, \mu)=Q(s, \mu), P^{\prime}(s, \mu)=Q^{\prime}(s, \mu)
$$

and $P^{\prime \prime}(s, \mu) \neq Q^{\prime \prime}(s, \mu)$, concretely,

$$
\begin{aligned}
& w_{44} w_{42}^{-1} \delta^{-1} \boldsymbol{M}_{4} \mu s-\delta^{-1} w_{14} \boldsymbol{M}_{1} \mu \\
& =\left(1+w_{14} w_{33} w_{31}^{-1}\right) s^{\frac{\lambda_{1}}{\rho_{1}}}-\delta^{-1} \delta_{u} w_{14} s^{\frac{\lambda_{2}}{\rho_{1}}}+\text { h.o.t., } \\
& w_{44} w_{42}^{-1} \delta^{-1} \boldsymbol{M}_{4} \mu=\frac{\lambda_{1}}{\rho_{1}}\left(1+w_{14} w_{33} w_{31}^{-1}\right) s^{\frac{\lambda_{1}}{\rho_{1}}-1} \\
& -\frac{\lambda_{2}}{\rho_{1}} \delta^{-1} \delta_{u} w_{14} s^{\frac{\lambda_{2}}{\rho_{1}}-1}+\text { h.o.t., } \\
& 0 \neq \lambda_{1}\left(\lambda_{1}-\rho_{1}\right)\left(1+w_{14} w_{33} w_{31}^{-1}\right) s^{\frac{\lambda_{1}}{\rho_{1}}-2} \\
& -\lambda_{2}\left(\lambda_{2}-\rho_{1}\right) \delta^{-1} \delta_{u} w_{14} s^{\frac{\lambda_{2}}{\rho_{1}}-2}+\text { h.o.t.. }
\end{aligned}
$$

Then the tangent point

$$
s^{*}=\left(\frac{\rho_{1} w_{44} M_{4} \mu}{\left(1+w_{14} w_{33} w_{31}^{-1}\right) \lambda_{1} \delta w_{42}}\right)^{\frac{\rho_{1}}{\lambda_{1}-\rho_{1}}}+\text { h.o.t. }
$$

as $w_{42} w_{44} \boldsymbol{M}_{4} \mu>0$. Combining the first equation of (3.2) 
with the tangent point, we obtain the double periodic orbit bifurcation surface

$$
\begin{aligned}
& S N^{1}: w_{14} \boldsymbol{M}_{1} \mu=\frac{\left(\lambda_{1}-\rho_{1}\right) w_{44}}{\lambda_{1} w_{42}} \\
& \cdot\left(\frac{\rho_{1} w_{44}}{\left(1+w_{14} w_{33} w_{31}^{-1}\right) \lambda_{1} \delta w_{42}}\right)^{\frac{\rho_{1}}{\lambda_{1}-\rho_{1}}}\left(\boldsymbol{M}_{4} \mu\right)^{\frac{\lambda_{1}}{\lambda_{1}-\rho_{1}}}+\text { h.o.t. }
\end{aligned}
$$

in the region $\mu \in R_{22}$. At the same time, when $\mu \in R_{22}$, the line $W=P(s, \mu)$ lies under the curve $W=Q(s, \mu)$, see Figure 2(c), so if $-w_{14} \boldsymbol{M}_{1} \mu$ increases, the line must intersects the curve at two sufficiently small positive points, therefore system (1.1) undergos two 1-periodic orbits. Then the proof is complete.

Theorem 3.3 Suppose that $\operatorname{Rank}\left(\boldsymbol{M}_{1}, \boldsymbol{M}_{4}\right)=2$, then system (1.1) has only one 1-homoclinic orbit near $\Gamma$ in the region $R_{01}$; has only one 1-periodic orbit near $\Gamma$ in the region $R_{01}$; has exactly one 1-homoclinic orbit and one 1-periodic orbit near $\Gamma$ in the region $R_{02}$; has not any 1-periodic orbit or 1-homoclinic orbit in the region $R_{20}$.

Proof When

$$
\begin{gathered}
\mu \in R_{02}=\left\{\mu \mid w_{14} \boldsymbol{M}_{1} \mu=0, w_{42} w_{44} \boldsymbol{M}_{4} \mu>0\right\}, \\
F(s, \mu)=s\left(\left(1+w_{14} w_{33} w_{31}^{-1}\right) s^{\frac{\lambda_{1}}{\rho_{1}}-1}+\delta^{-1} w_{14} \boldsymbol{M}_{3} \mu s^{\frac{\lambda_{2}}{\rho_{1}}-1}\right. \\
\left.-w_{44} w_{42}^{-1} \delta^{-1} \boldsymbol{M}_{4} \mu-w_{44} w_{42}^{-1}\left(w_{32}-w_{12} w_{33}\right) w_{31}^{-1} s^{\frac{\lambda_{1}}{\rho_{1}}}+\text { h.o.t. }\right) \\
=0
\end{gathered}
$$

has always two solutions $s_{1}=0$ and

$$
S_{2}=\left(\frac{w_{44} \boldsymbol{M}_{4} \mu}{\left(1+w_{14} w_{33} w_{31}^{-1}\right) w_{42} \delta}\right)^{\frac{\rho_{1}}{\lambda_{1}-\rho_{1}}}+\text { h.o.t. }
$$

or has only a zero solution $s_{1}=0$ for

$$
\mu \in R_{01}=\left\{\mu \mid w_{14} \boldsymbol{M}_{1} \mu=0, w_{42} w_{44} \boldsymbol{M}_{4} \mu<0\right\} .
$$

While for

$$
\mu \in R_{10}=\left\{\mu \mid w_{14} \boldsymbol{M}_{1} \mu<0, w_{42} w_{44} \boldsymbol{M}_{4} \mu=0\right\},
$$

apparently the line $W=P(s, \mu)$ is horizontal. So $F(s, \mu)=0$ gives merely a solution

$$
s_{0}=\left(-\frac{w_{14} \boldsymbol{M}_{1} \mu}{\left(1+w_{14} w_{33} w_{31}^{-1}\right) \delta}\right)^{\frac{\rho_{1}}{\lambda_{1}}}+\text { h.o.t. . }
$$

The last conclusion is obvious for

$$
\mu \in R_{20}=\left\{\mu \mid w_{14} \boldsymbol{M}_{1} \mu>0, w_{42} w_{44} \boldsymbol{M}_{4} \mu=0\right\} .
$$

In the following, we study the case $w_{44}=0$. Then (3.1) is

$$
\begin{aligned}
& F(s, \mu)=\left(1+w_{14} w_{33} w_{31}^{-1}\right) s^{\frac{\lambda_{1}}{\rho_{1}}}+\delta^{-1} w_{14} \boldsymbol{M}_{3} \mu s^{\frac{\lambda_{2}}{\rho_{1}}} \\
& +\delta^{-1} w_{14} \boldsymbol{M}_{1} \mu-w_{14} w_{43} w_{42}^{-1} \delta^{-1} \boldsymbol{M}_{4} \mu s^{\frac{\rho_{1}+\lambda_{2}}{\rho_{1}}}+\text { h.o.t. }=0 .
\end{aligned}
$$

Similar to the proof of Theorem 3.1 and 3.3, we have

Theorem 3.4 Suppose that $\operatorname{Rank}\left(\boldsymbol{M}_{1}, \boldsymbol{M}_{4}\right)=2$, then in the region $D_{21} \cup D_{12}$, system (1.1) has a unique 1-periodic orbit near $\Gamma$; in the region $D_{22}$, system (1.1) has not any 1-periodic orbit.

Proof Redefine

$$
\begin{aligned}
P(s, \mu) & =-\delta^{-1} w_{14} \boldsymbol{M}_{3} \mu s^{\frac{\lambda_{2}}{\rho_{1}}}-\delta^{-1} w_{14} \boldsymbol{M}_{1} \mu+\text { h.o.t., } \\
Q(s, \mu) & =\left(1+w_{14} w_{33} w_{31}^{-1}\right) s^{\frac{\lambda_{1}}{\rho_{1}}} \\
& -w_{14} w_{43} w_{42}^{-1} \delta^{-1} \boldsymbol{M}_{4} \mu s^{\frac{\rho_{1}+\lambda_{2}}{\rho_{1}}}+\text { h.o.t. }
\end{aligned}
$$

By studying the relationship between the curves $f=P(s, \mu)$ and $f=Q(s, \mu)$, it is easy to get the main ideas, see Figure 3. Here

$$
\begin{aligned}
& D_{12}=\left\{\mu \mid w_{14} \boldsymbol{M}_{1} \mu<0, w_{14} \boldsymbol{M}_{3} \mu>0\right\}, \\
& D_{21}=\left\{\mu \mid w_{14} \boldsymbol{M}_{1} \mu>0, w_{14} \boldsymbol{M}_{3} \mu<0\right\}
\end{aligned}
$$

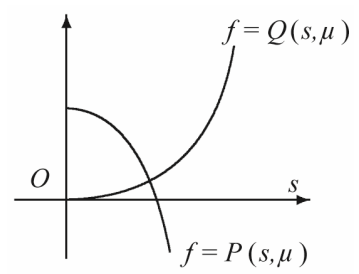

(a)

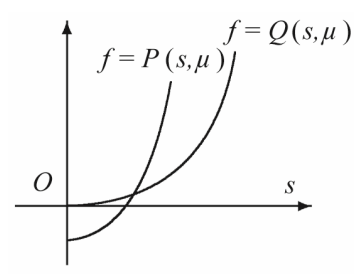

(b)

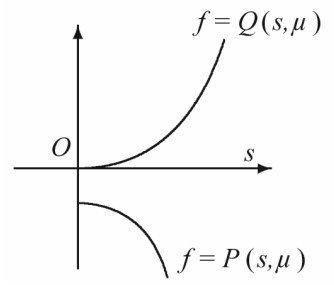

(c)

Figure 3. Location between the curves. (a) $\mu \in D_{12}$; (b) $\mu \in$ $D_{21}$; (c) $\mu \in D_{22}$. 
and

$$
D_{22}=\left\{\mu \mid w_{14} \boldsymbol{M}_{1} \mu>0, w_{14} \boldsymbol{M}_{3} \mu>0\right\} .
$$

Remark 3.1 For the case $w_{44}=0$, system (1.1) has no longer double 1-periodic orbits and the double 1-periodic orbit bifurcation surfaces.

Theorem 3.5 Suppose that $\operatorname{Rank}\left(\boldsymbol{M}_{1}, \boldsymbol{M}_{4}\right)=2$, then system (1.1) has only one 1-homoclinic orbit near $\Gamma$ in the region $D_{0}$; has only one 1-periodic orbit near $\Gamma$ in the region $D_{10}$; has not any 1-periodic orbit or 1-homoclinic orbit in the region $D_{20}$.

Proof Notice that

$$
\begin{aligned}
& F(s, \mu)=s\left(\left(1+w_{14} w_{33} w_{31}^{-1}\right) s^{\frac{\lambda_{1}}{\rho_{1}}-1}+\delta^{-1} w_{14} \boldsymbol{M}_{3} \mu s^{\frac{\lambda_{2}}{\rho_{1}}-1}\right. \\
& \left.-w_{14} w_{43} w_{42}^{-1} \delta^{-1} \boldsymbol{M}_{4} \mu s^{\frac{\lambda_{2}}{\rho_{1}}}+\text { h.o.t. }\right)=0
\end{aligned}
$$

has only a zero solution $s_{1}=0$ for

$$
\mu \in D_{0}=\left\{\mu \mid w_{14} M_{1} \mu=0\right\} .
$$

And the line $f=P(s, \mu)$ is horizontal for

$$
\begin{aligned}
& \mu \in D_{10}=\left\{\mu \mid w_{14} \boldsymbol{M}_{1} \mu<0, w_{14} \boldsymbol{M}_{3} \mu=0\right\} \cup D_{20} \\
& =\left\{\mu \mid w_{14} \boldsymbol{M}_{1} \mu>0, w_{14} \boldsymbol{M}_{3} \mu=0\right\} .
\end{aligned}
$$

Thereby the conclusion is clear. We omit the details here.

\section{Conclusion}

The theoretical development of flip bifurcations indeed advanced much in recent years. More and more complicated cases with several flips or accompanied by transcritical bifurcation nowadays are discussed. This paper focuses on a kind of three flips homoclinic case with resonance and introduces an effective method to extend the study. By the analysis of the bifurcation equation, the existence of a double 1-periodic orbit, some 1-periodic orbits and 1-homoclinic orbits, and the coexistence conditions of 1-periodic orbits and 1-homoclinic orbits are given. From the study, one notice that different leading terms of the bifurcation equation may cause different bifurcation phenomena, so we can go further in the future work.

\section{REFERENCES}

[1] A. J. Homburg and B. Krauskopf, "Resonant Homoclinic Flip Bifurcations,” Journal of Dynamics and Differential Equations, Vol. 12, No. 4, 2000, pp. 807-850. doi:10.1023/A:1009046621861

[2] A. J. Homburg, H. Kokubu and M. Krupa, "The Cusp Horseshoe and Its Bifurcations in the Unfolding of an Inclinication-Flip Homoclinic Orbit,” Ergodic Theory and
Dynamical Systems, Vol. 14, No. 4, 1994, pp. 667-693. doi:10.1017/S0143385700008117

[3] B. E. Oldeman, B. Krauskopf and A. R. Champneys, "Numerical Unfoldings of Codimension-Three Resonant Homoclinic Flip Bifurcations,” Nonlinearity, Vol. 14, No. 3, 2001, pp. 597-621. doi:10.1088/0951-7715/14/3/309

[4] C. A. Morales and M. J. Pacifico, "Inclination-Flip Homoclinic Orbits Arising from Orbit-Flip,” Nonlinearity, Vol. 14, No. 2, 2001, pp. 379-393. doi:10.1088/0951-7715/14/2/311

[5] E. Catsigeras and H. Enrich, "Homoclinic Tangencies Near Cascades of Period Doubling Bifurcations,” Annales de l'Institut Henri Poincare (C) Non Linear Analysis, Vol. 15, No. 3, 1998, pp. 255-299. doi:10.1016/S0294-1449(98)80119-4

[6] H. Kokubu, M. Komuru and H. Oka, "Multiple Homoclinic Bifurcations from Orbit Flip I. Sucessive Homoclinic Doublings," International Journal of Bifurcation and Chaos, Vol. 6, No. 5, 1996, pp. 833-850. doi:10.1142/S0218127496000461

[7] J. A. Yorke and K. T. Alligood, "Cascades of Period Doubling Bifurcations: A Prerequisite for Horseshoes," Bulletin of the American Mathematical Society, Vol. 9, 1983, pp. 319-322. doi:10.1090/S0273-0979-1983-15191-1

[8] M. V. Shashkov and D. V. Turaev, “An Existence Theorem of Smooth Nonlocal Center Manifolds for Systems Close to a System with a Homoclinic Loop," Journal of Nonlinear Science, Vol. 9, No. 5, 1999, pp. 525-573. doi:10.1007/s003329900078

[9] M. Kisaka, H. Kokubu and H. Oka, "Supplement to Homoclinic-Doubling Bifurcation in Vector Fields," Dynamical Systems, Longman, London, 1993, pp. 92-116.

[10] M. Kisaka, H. Kokubu and H. Oka, "Bifurcations to NHomoclinic Orbits and N-Periodic Orbits in Vector Fields," Journal of Dynamics and Differential Equations, Vol. 5, No. 2, 1993, pp. 305-357. doi:10.1007/BF01053164

[11] F. Geng and D. Zhu, "Bifurcations of Generic Heteroclinic Loop Accompanied by Transcritical Bifurcation," International Journal of Bifurcation and Chaos, Vol. 18, No. 4, 2008, pp. 1069-1083. doi:10.1142/S0218127408020847

[12] Q. Lu, Z. Qiao, T. Zhang and D. Zhu, "Heterodimensional Cycle Bifurcation with Orbit-Filp," International Journal of Bifurcation and Chaos, Vol. 20, No. 2, 2010, pp. 491-508. doi:10.1142/S0218127410025569

[13] X. Liu, "Homoclinic Flip Bifurcations Accompanied by Transcritical Bifurcation,” Chinese Annals of Mathematics, Series B, Vol. 32, No. 6, 2011, pp. 905-916. doi:10.1007/s11401-011-0675-y

[14] T. Zhang and D. Zhu, "Homoclinic Bifurcation of Orbit Flip with Resonant Principal Eigenvalues,” Acta Mathematica Sinica, Vol. 22, No. 3, 2006, pp. 855-864.

[15] T. Zhang and D. Zhu, "Bifurcations of Homoclinic Orbit Connecting Two Nonleading Eigendirections,” International Journal of Bifurcation and Chaos, Vol. 17, No. 3, 2007, pp. 823-836. doi:10.1142/S0218127407017574 